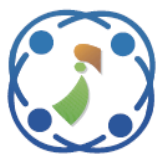

\title{
An Efficient Ultrasonic Method to Control the Coagulation Enzymatic of Milk
}

\author{
Firdaous Bakkali $^{1}$, Mourad Derra ${ }^{1 *}$, Abdellah Amghar ${ }^{1}$, Hassan Sahsah ${ }^{1}$ \\ ${ }^{1}$ Laboratory of Metrology and Information Treatment, College of Science, \\ Ibn Zohr University, BP 8106, 80000 Agadir, Morocco \\ * Corresponding author's Email: mourad.derra@edu.uiz.ac.ma
}

\begin{abstract}
A non-destructive ultrasonic technique presented in this paper to monitor in real time the renneted milk coagulation process in order to predict the clotting time. This one is often used as a reference to determine the cutting time of the gel, which means that the whey trapped in the gel pores has been expelled. During the enzymatic coagulation of milk, the change of the flight time has been exploited to determine the clotting time with high precision. The effects of the temperature (Standard temperature (ST), High temperature (HT) and Low temperature (LT)) and rennet concentration (Standard rennet (SR), High rennet (HR) and Low rennet (LR)) on the enzymatic coagulation were studied.
\end{abstract}

Keywords: Ultrasonics, Control non-destructive, Enzymatic coagulation, Time of flight (TOF), Clotting time.

\section{Introduction}

In the manufacture of cheese, the most important consideration is to obtain an acceptable product from the point of view of flavor and texture. The texture, flavor, and aroma of the product are related to composition, $\mathrm{pH}$ of the curd and the ripening conditions. Since the curd characteristics depend on those of the coagulum, the first step, coagulation, plays an important role in determining the end product qualities. Hence, the major concern of both researchers and cheesemakers is about mastering and controlling this step of cheese production as well as possible. In the majority of cheese varieties manufacture, milk proteins are coagulated to form a continuous solid curd in which milk fat globules, water and water-soluble materials are entrapped. Enzymatic coagulation is induced by microbiallyproduced enzymes, or by an enzyme extract from calf stomachs (rennet). These enzymes begin coagulation of milk by cleaving and hydrolyzing kcasein. This later leads to destabilizing the colloidal system followed by protein micelles aggregation into clusters. The clusters grow, then the chains cross-link and transform milk into a gel. As a result of the coagulation process, a certain curd firmness has been reached. The gel is then sliced into approximating $7 \mathrm{~mm}$ cubes. The coagulating matrix shrinks and forces liquid from the cubes (syneresis) resulting in a two-phase system of curd and whey. After being separated from the whey, the curd is further processed into a cheese product.

In practice, gel is usually cut upon the operator's judgment based on subjective evaluation of textural and visual gel properties or after a predetermined reaction time. This later is a very common practice, however, it is not precise due to the variation of the factors that affect curd firmness and gel microstructure which will change the optimum cutting time. if the evaluation is made properly, cutting the curd by relying on empirical inspection is accurate and acceptable. Cheese makers can select the cutting time with amazing consistency [1], but it is risky to assume that curd firming and gel tendency to exhibit syneresis can be "optimized" by experimental testing. The lack of a rigorous cutting time characterization and the usual changes in milk protein content are forcing modern cheese plants to find other methods in order to control coagulation, gel firming, curd syneresis, cheese yield, and product quality [2-4]. 
Several studies aimed at controlling in real time the process of milk coagulation were carried out to determine the clotting time and therefore the perfect time for slicing the gel [5-20]. However, most of these methods have a destructive character that their direct contact with the coagulum causes the deformation of the gel what limits the quality of the final product. To find more effective ways having a non-destructive nature, then the use of ultrasound proves interesting [21-28]. Because of their ease of placing in-situ and their non destructive properties, the techniques of ultrasonic wave propagation seem particularly well suited to monitor the gelation of the milk. Ultrasonic velocity and attenuation have become very valuable tools for studying the physical properties of matter. For this reason, many studies on milk coagulation are based on the monitoring of these two quantities (velocity and attenuation) during the clotting process using the technique of transmission. However, these studies use only the attenuation evolution to determine the clotting time. Using an ultrasonic resonator technique [29] measured with a good resolution the velocity of phase and attenuation during coagulation. However, this technique has not been used to determine the clotting time. Another method, our research group, [24] use an ultrasonic pulse technique by reflection for determining the clotting time from the phase velocity changing by calculating the second derivative of the polynomial function obtained by smoothing the experimental curve of the phase velocity.

In this work, we have developed a nondestructive ultrasonic technique for monitoring changes in the time of flight during the enzymatic coagulation of milk. Our proposed method has advantages over many traditional analytical techniques since it provides non-destructive, rapid and cheap measurements. Furthermore, it includes just one transducer and it is non-invasive, which is very important for hygiene reasons in food industry. The rest of the paper is organized as follows: The materials and methods are described in section 2 . The measurement technique is described in details in section 3. Results and discussion are given in section 4. Conclusion is drawn in section 5 .

\section{Materials and Methods}

\subsection{Materials}

We conducted our experiments with skim milk powder from the same package, purchased commercially. For $90 \mathrm{~g}$ of water, we added $13 \mathrm{~g}$ of skimmed milk powder. Everything is highly homogenized and placed in a thermostatted water bath at the desired temperature for 30 minutes before being rennet. The rennet used is sold in pharmacies under the name Caille-lait universel $0.22 \mathrm{~g} / \mathrm{l}$, a product of society COOPER Morocco. Rennet tablet is dissolved separately in a little water. The proportions recommended by the manual rennet have been respected; the equivalent of one tablet per liter of reconstituted milk. Homogenization of the whole milk + rennet is necessary to avoid creating bubbles. The acquisition of the signal received by the transducer at every step of one minute is launched on PC. The data is then processed in the LabVIEW program in order to determinate the viscoelastic parameters of milk. As the temperature and the concentration of rennet are the main factors affecting the enzymatic phase of coagulation of milk three levels of each of these parameters were considered during the experiments. They represent a standard level and two levels, one is below and the other is above (see Table 1).

\subsection{Ultrasonic system}

The Fig. 1 shows the device of measurement used. A center frequency transducer of $5 \mathrm{MHz}(0,5 \mathrm{in}$, crystal diameter, A309S-SU Model, Panametrics, Olympus) immersed in water, is used to make the ultrasonic impulse crosses the container enclosing the milk sample. The whole is in a thermostat tank. The sensor is connected to a generator pulse (Sofranel Model 5073PR, Sofranel Instruments) which plays the role of transmitter/receiver that sends the electric signal. The received signal after interfaces reflections: water / Plexiglas, Plexiglas / milk and milk / Plexiglas, is amplified and digitized by a PicoScope. The different treatments applied to digital signal obtained are performed using a LabVIEW program, developed in this work and implemented on a personal computer. The incident signal follows the path shown by the diagram of Fig. 2.

Table 1. Experimental Conditions

\begin{tabular}{|c|c|c|}
\hline Conditions & $\begin{array}{c}\text { Temperature } \\
{ }^{\circ} \mathbf{C}\end{array}$ & $\begin{array}{c}\text { Rennet } \\
\text { concentration (g/l) }\end{array}$ \\
\hline SR/ST & 37 & 0.22 \\
\hline LR/ST & 37 & 0.20 \\
\hline HR/ST & 37 & 0.24 \\
\hline SR/LT & 32 & 0.22 \\
\hline SR/HT & 42 & 0.22 \\
\hline
\end{tabular}




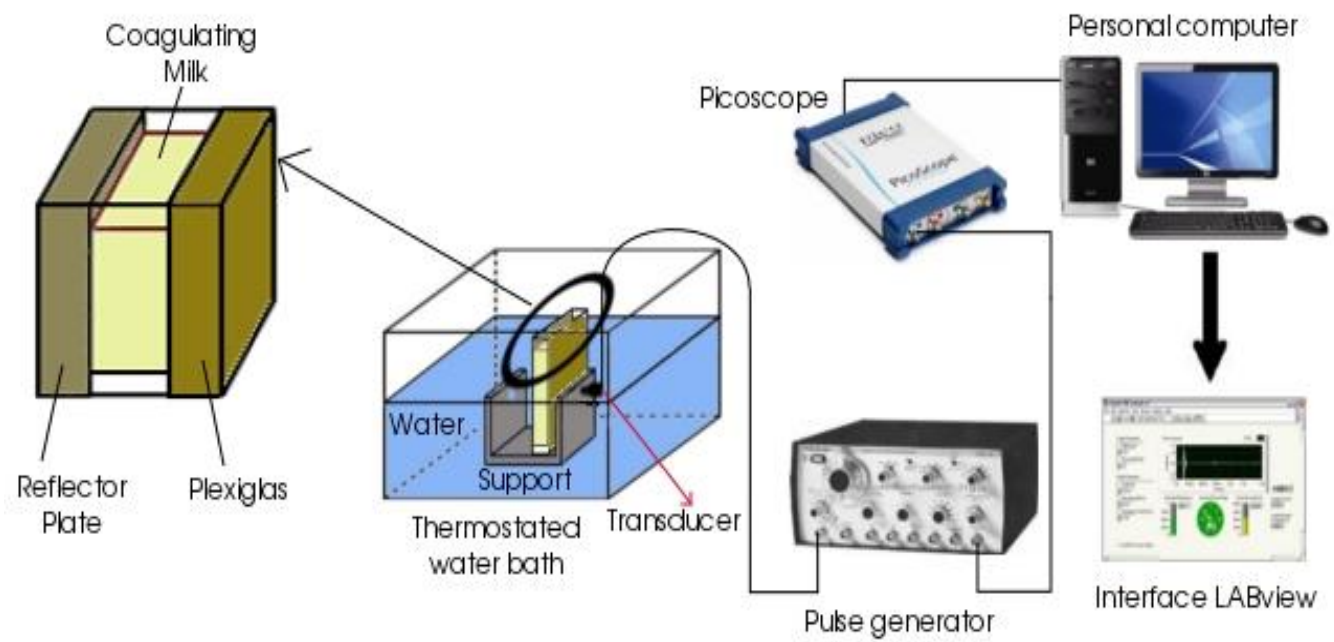

Figure.1 Experimental set-up

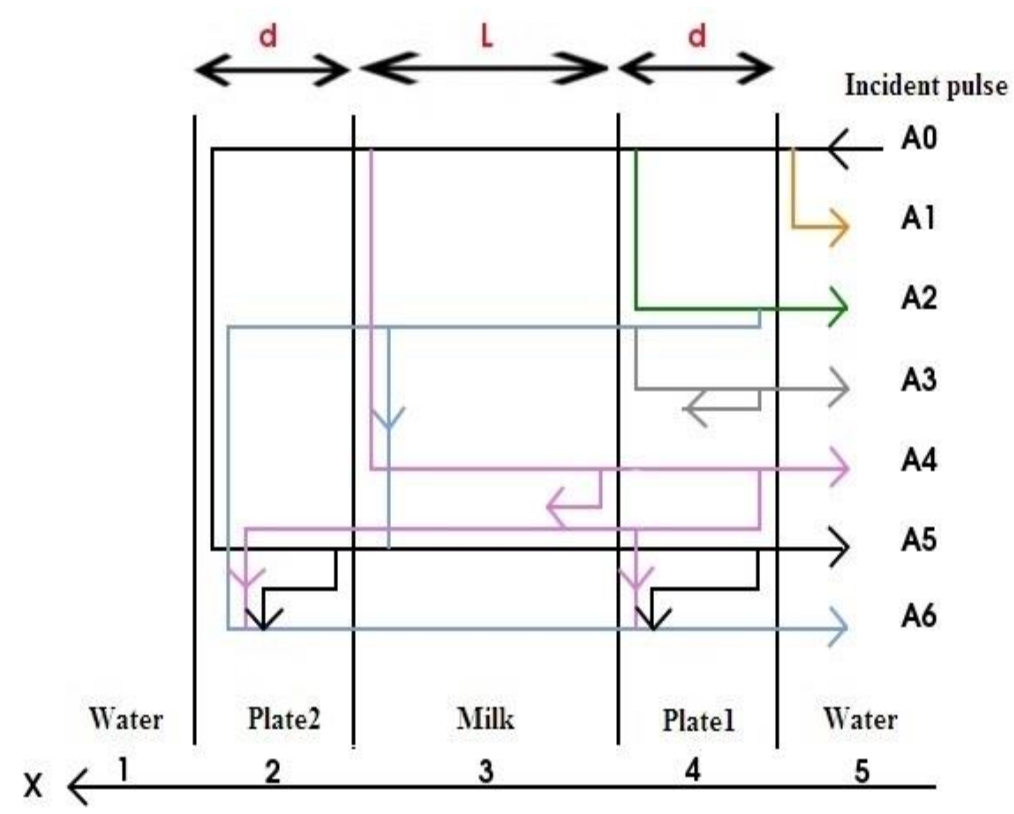

Figure.2 Different paths of propagation

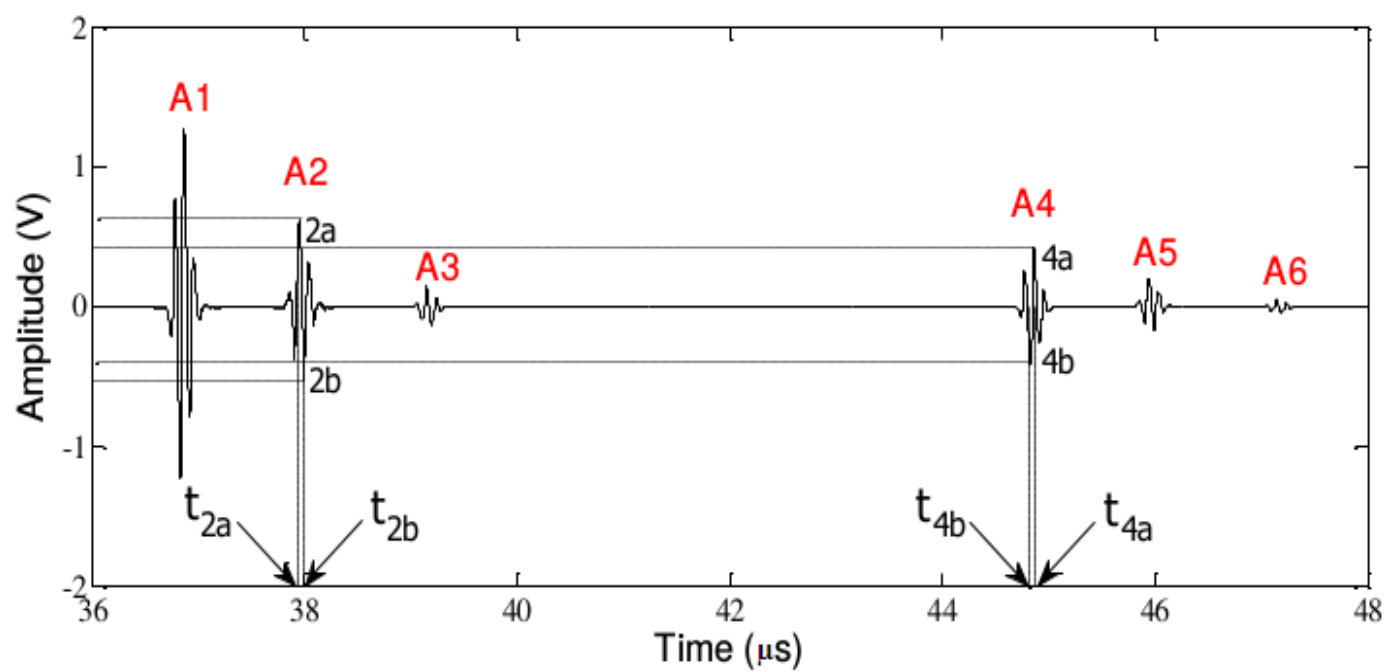

Figure.3 Type of the signal reflected by the container enclosing milk 


\subsection{Control in real time under LabVIEW interface of coagulating milk}

We start the control by acquiring the formed signals on the computer based on an application programmed with LabVIEW language. The realized program allows us to specify the number of wanted acquisitions during the experiment and the time between each other. This program captures each time 50 signals and realizes an averaging to neutralize noised signals. The resulting signal represents for the user a single acquisition (Fig. 3).

This signal comprises a first part composed of three echoes:

- Echo A1, linked to the specular reflection of the incident beam at the interface between water and the first face of the plate 1 ,

- The second echo A2 corresponding to the reflection at the interface between the second side of the plate 1 and the enclosed milk,

- The third echo A3 corresponding to the second back and forth in the plate 1 .

The second part includes also three echoes, having traversed all the trapped milk in back and forth:

- The first echo A4 corresponds to reflection at the interface between the milk and the plate 2,

- The echo A5 represents a superposition of two echoes: one make a back and forth in the plate 1 , and the other one in the plate 2 ,

- The third echo of the series A6 corresponds to a superposition of three echoes: an echo which made two back and forth to the plate 2 , an echo that made two back and forth to the plate 1 , and an echo corresponding to a back and forth in the plate 1 followed by a back and forth in the plate2.

\section{Measurement technique}

The used ultrasonic technique is about following in real time the evolution of the phase velocity and the time of flight. Determining viscoelastic parameters in the case of plate immersed in liquid has been the subject of several studies [30-34], allows us to obtain the viscoelastic parameters of the milk enclosed in a cube container. This determination is made by analyzing the time signals experimentally obtained (Fig. 3).

The expression of an ultrasonic wave can be written in the integral form:

$$
\begin{aligned}
f(x, t) & =\int_{-\infty}^{+\infty} A_{0}(v) \exp [-\alpha(v) x] \\
& \exp \left[-j 2 \pi v\left(t-\frac{x}{V}\right)\right] d v
\end{aligned}
$$

Where $A_{0}(v)$ is the original amplitude for the frequency $v, \alpha(v)$ is the coefficient of attenuation due to the viscoelasticity, $x$ is the distance covered by the wave, $t$ is the time and $V$ is the phase velocity in the material. The Fourier transform of this response is:

$$
F(x, v)=A_{0}(v) \exp [-\alpha(v) x] \exp \left[-j 2 \pi v \frac{x}{V}\right]
$$

This is calculated again for each echo of figure 3 and each medium interface.

\subsection{Phase velocity}

R.A.Kline [30] and D.Mak [31,34] have used similar methods to measure the phase velocity in a plate with a contact transducer, the coupling between the plate and the transducer is realized with a thin layer of acoustic gel. For the measurement of the phase velocity in the milk, the factors containing the phase are introduced. The Fourier Transform of the two echoes $\mathrm{A}_{2}$ and $\mathrm{A}_{4}$ will have the complex expression:

$$
\begin{aligned}
A_{2}= & A_{0}(v) T_{54} R_{43} T_{45} \exp \left[-2 \alpha_{p g}(v) d\right] \\
& \exp j\left[\frac{\omega}{V_{p g}} 2 d+\phi_{0}\right] \\
A_{4}= & A_{0}(v) T_{54} T_{43} R_{32} T_{34} T_{45} \\
& \exp \left[-2 \alpha_{p g}(v) d\right] \exp \left[-2 \alpha_{\text {milk }}(v) L\right] \\
& \exp j\left[\frac{\omega}{V_{p g}} 2 d+\frac{\omega}{V_{\text {milk }}} 2 L+\phi_{0}\right]
\end{aligned}
$$

where $V_{p g}$ and $V_{\text {milk }}$ are the phase velocities in the Plexiglas and in the milk respectively, $\phi_{0}$ is the phase of the incident wave and $T_{i j}$ (respectively $R_{i j}$ ) is the transmission coefficient (respectively the reflexion coefficient) between mediums $i$ and $j$.

The phases of the echoes $A_{2}$ and $A_{4}$ are given by :

$$
\phi_{p g / m i l k}=\frac{\omega}{V_{p g}} 2 d+\phi_{0}
$$




$$
\phi_{\text {milk/pg }}=\frac{\omega}{V_{p g}} 2 d+\frac{\omega}{V_{\text {milk }}} 2 L+\phi_{0}
$$

The phase velocity is then equal to :

$$
V_{\text {milk }}(v)=\frac{2 \omega L}{\phi_{\text {milk/pg }}-\phi_{p g / m i l k}}
$$

With $\omega=2 \pi \nu$

The Fourier transform of the echo $A_{2}$ isolated using the temporal filtering program LabVIEW gives the real parts $R_{2}(\omega)$ and imaginary $I_{2}(\omega)$ which let us compute the phase $\phi_{p g / m i l k}$ according to the classic equation:

$$
\phi_{p g / m i l k}=\arctan \left(\frac{I_{2}(\omega)}{R_{2}(\omega)}\right)
$$

While the Fourier transform of the echo $A_{4}$ gives us isolated real parts $R_{4}(\omega)$ and imaginary parts $I_{4}(\omega)$ which let us compute the phase $\phi_{\text {milk/pg }}$ :

$$
\phi_{\text {milk/pg }}=\arctan \left(\frac{I_{4}(\omega)}{R_{4}(\omega)}\right)
$$

In the calculation of these two phases, we obtain two spectrums whose phase varies between $\pi / 2$ and $-\pi / 2$. However, it is necessary to have a continuous phase spectrum in order to obtain the phase velocity. That is why we have designed an algorithm to place the phases in continuous form. Therefore the knowledge of $\phi_{p g / m i l k}$ and $\phi_{\text {milk/pg }}$ can be traced back to the phase velocity $V_{\text {milk }}$ in the milk enclosed in its container.

\subsection{Time of flight}

The flight time technique consists of measuring the propagation time of a pulse, sent by the transducer to its return on the same transducer (transmitter / receiver), through the milk.

The time of flight is written as in the following formula:

$$
T O F=1 / 2\left[\left(t_{4 a}-t_{2 a}\right)+\left(t_{4 b}-t_{2 b}\right)\right]
$$

\section{Results and Discussion}

Ultrasonic techniques developed until today to determine the clotting time, are based on the evolution of the phase velocity. The latter, as it was used by [24] consists of using the second derivative of the phase velocity and consider the extreme of this second derivative as the transition point which marks the end of the enzymatic phase of coagulation. This transition point has been identified as clotting time [23].

In this work, we applied this method for determining the clotting time in our experimental results, and we compared it to our proposed method based on the exploitation of the flight time. The Fig. 4 presents, in our standard experimental conditions, the change as a function time of the smoothed time of flight and its second derivative. The time of flight evolution is done in two steps. The first step represents a linearly and rapidly decreasing straight and the second step represents a linearly and slowly decreasing straight. This decrease can be interpreted as a change in the mechanical strength of the milk.

The clotting time is then evaluated as the intersection between the line representing the first step and the line representing the second step. This intersection coincides with the first derivative inflection point of the time of flight. Because it is difficult to localize the point of intersection between the two straights, it is more practical to use the inflection point of the time of flight first derivative (to find the first derivative inflection point it was obligatory to compute the extremum of the second derivative of the time of flight). Since the clotting time is defined as the transition point between the two steps of time of flight evolution, this point can be found by considering the extremum of the second derivative of the time of flight.

It should be noted that in the previous method the calculation of phases used to compute the phase velocity, two spectra whose phase varies between $\pi / 2$ and $-\pi / 2$ are obtained, however, it is necessary to achieve continuous phase spectra for the calculation of the speed of phase. Which required unwrapping the phases into a continuous form. This unwrapping needs a signal that contains a huge number of values.

The values of the clotting time measured from the second derivative of flight time and phase velocity are grouped for the different experimental conditions in Table 2 . The time of flight obtained at different rennet concentrations and temperatures is shown in Fig. 5 and Fig. 6.

A total of 15 experiments were conducted in the laboratory, three repetitions at least were performed for every experimental condition. The standard error $\Delta P_{t}$ was obtained using the equation: 


$$
\Delta P_{t}=\frac{\sigma}{\sqrt{n}}
$$

Where $n$ is the number of experiments and $\sigma$ is the standard deviation.

Note that the transition point at an elevated temperature $\left(42^{\circ} \mathrm{C}\right)$ is earlier than in the case of the standard temperature $\left(37^{\circ} \mathrm{C}\right)$. The same applies to a high rennet concentration, the transition point is earlier than in the case of a standard concentration. This indicates that the coagulation is much faster than the temperature or the rennet concentration are high.

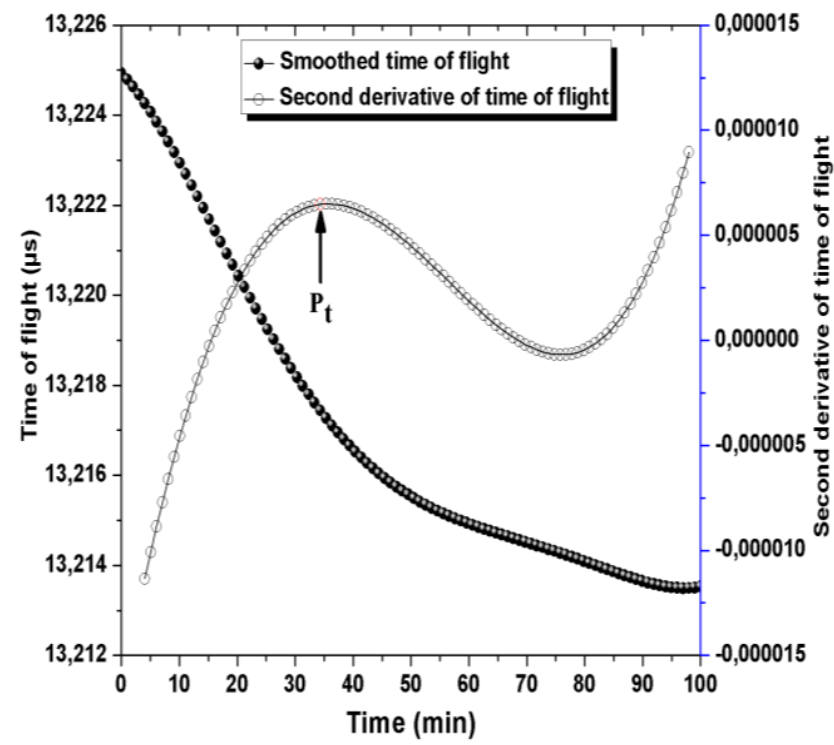

Figure.4 Identification of the transition point from the second derivative of the time of flight under standard conditions

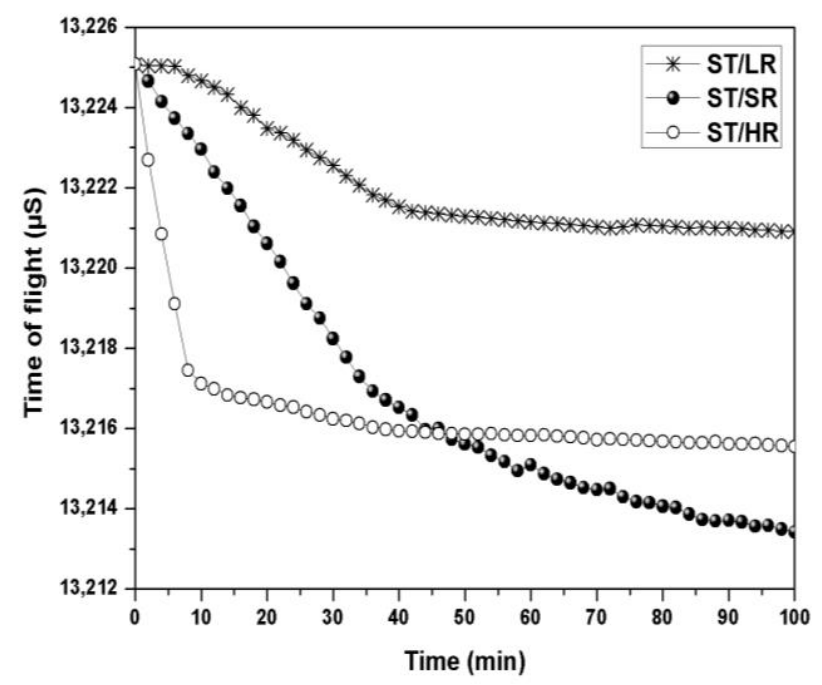

Figure.5: Time of flight through milk coagulation at different rennet concentration

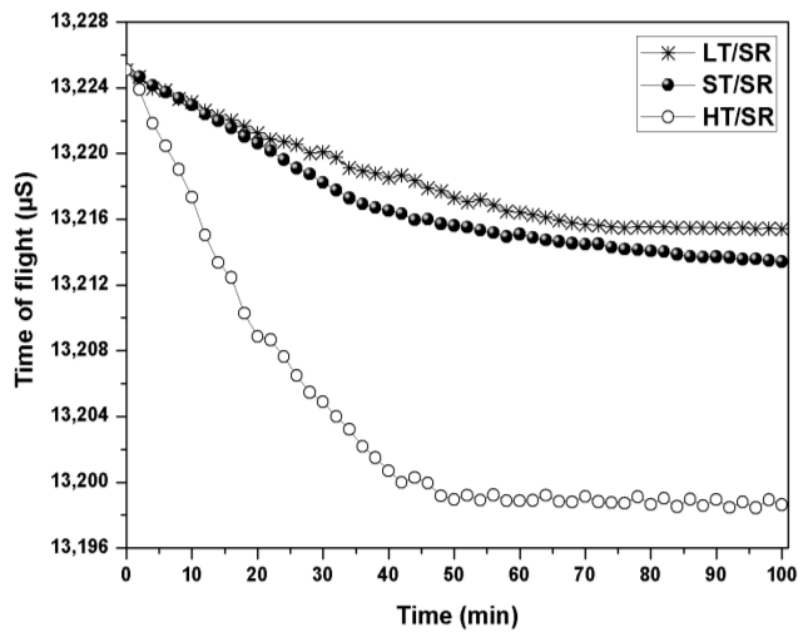

Figure.6: Time of flight through milk coagulation at different temperature

Table 2. Clotting time measured for the different experimental conditions of temperature and rennet concentration compared with the phase velocity method

\begin{tabular}{|c|c|c|}
\hline Conditions & Phase velocity & Time of flight \\
\hline SR/ST & $35.40 \pm 0.93 \mathrm{~min}$ & $34.95 \pm 1.04 \mathrm{~min}$ \\
\hline LR/ST & $38.50 \pm 1.68 \mathrm{~min}$ & $35.86 \pm 0.45 \mathrm{~min}$ \\
\hline HR/ST & $24.70 \pm 1.04 \mathrm{~min}$ & $24.26 \pm 1.02 \mathrm{~min}$ \\
\hline SR/LT & $39.81 \pm 1.40 \mathrm{~min}$ & $39.38 \pm 1.83 \mathrm{~min}$ \\
\hline SR/HT & $27.45 \pm 0.99 \mathrm{~min}$ & $27.51 \pm 1.02 \mathrm{~min}$ \\
\hline
\end{tabular}

\section{Conclusion}

The new ultrasound technique developed in this work allows us to determine the clotting time, based on time of flight changes. Owing to the simplicity of its conditions (without unwrapping), this technique may replace the phase velocity based method determining the clotting time. So this technique proves to be well suited for this kind of control: it is non destructive and non invasive, especially since it makes - using a program - a real time control. The obtained results of our proposed method are in agreement with the results obtained using the phase velocity coagulation time. More works should be done to implement our method in an embedded system that would be more applicable to on-line industrial measurements.

\section{Acknowledgements}

This work was supported and funded by the University Ibn Zohr.

\section{References}

[1] A.C.M. Van Hooydonk, and G. Van den Berg, "Control and determination of the curd setting 
during cheesemaking", IDF Bull, Vol.225, pp.210, 1988.

[2] D. G. Dalgleish, "The Enzymatic Coagulation of Milk" Cheese: Chemistry, Physics and Microbiology, Vol.1, General Aspects, pp.69100, 1993.

[3] D. B. Hyslop, "Enzymatic Coagulation of Milk", Advanced Dairy Chemistry, Proteins: Part A / Part B, pp. 839-878, 2003.

[4] M. Corredig, and E. Salvatore, "Enzymatic Coagulation of Milk", Advanced Dairy Chemistry, Vol.1B, Proteins: Applied Aspects, pp.287-307, 2016.

[5] M. Castillo, F. A. Payne, C. L. Hicks, M. B. Lpez, "Predicting cutting and clotting time of coagulating goats milk using diffuse reflectance: effect of $\mathrm{pH}$, temperature and enzyme concentration", International Dairy Journal, Vol.10, pp.551-562, 2000.

[6] S. Jovanovi, O. Maej, and J. D. Djurdjevi, "The influence of various factors on milk clotting time", Journal of Agricultural Sciences, Vol.47, pp.57-73, 2002.

[7] M. Castillo, F. A. Payne, C. L. Hicks, J. Laencina, M. B. Lpez, "Effect of protein and temperature on cutting time prediction in goats milk using an optical reflectance sensor", Journal of Dairy Research, Vol.70, pp.205-215, $2003 \mathrm{a}$.

[8] A. Lagaude, L. Fernandez, J. L. Cuq, and S. Marchesseau, "Characterization of curd formation during the rennet coagulation of milk by an optical microscopic method", International Dairy Journal, Vol.14, pp.10331039, 2004.

[9] M. Castillo, R. Gonzlez, F. A. Payne, J. Laencina, M. B. Lpez, "Optical monitoring of milk coagulation and inline cutting time prediction in Murcian al Vino cheese", Applied Engineering in Agriculture, Vol.21, pp.465-471, $2005 b$.

[10] M. Castillo, J. A. Lucey, F. A. Payne, "The effect of temperature and inoculum concentration on rheological and light scatter properties of milk coagulated by a combination of bacterial fermentation and chymosin. Cottage cheese-type gels", International Dairy Journal, Vol.15, pp.131-146, 2006b.

[11] C. C. Fagan, M. Castillo, C. P. ODonnell, D. J. OCallaghan, and F. A. Payne, "On-line prediction of cheese making indices using backscatter of near infrared light", International Dairy Journal, Vol.18, pp.120-128, 2008.

[12] M. J. Mateo, D. J. OCallaghan, C. D. Everard, M. Castillo, F. A. Payne, and C. P. ODonnell,
"Validation of a curd-syneresis sensor over a range of milk composition and process parameters", Journal of Dairy Science, Vol.92, pp.5386-5395, 2009b.

[13] N. Nicolau, M. Castillo, M. Buffa, D. J. OCallaghan, and B. Guamis, "Light scatter estimation of clotting and cutting time in sheep cheese manufacture", ICEF11, Athens, Greece, May 22-26, 2011.

[14] C. B. Lyndgaard, S. B. Engelsen, and F. W. J. Van den Berg, "Real-time modeling of milk coagulation using in-line near infrared spectroscopy", Journal of Food Engineering, Vol.108, pp.345-352, 2012.

[15] N. Tabayehnejad, M. Castillo, and F. Payne, "Comparison of total milk-clotting activity measurement precision using the Berridge clotting time method and a proposed optical method", Journal of Food Engineering, Vol.108, pp.549-556, 2012.

[16] M. De Marchi, V. Toffanin, M. Cassandro, and M. Penasa, "Prediction of coagulating and non coagulating milk samples using mid-infrared spectroscopy", Journal of Dairy Science, Vol.96, pp.4707-4715, 2013.

[17] O. Arango, M. Castillo, A. J. Trujillo, "Influence of fat replacement by inulin on rheological properties, kinetics of rennet milk coagulation and syneresis of milk gels". Journal of Dairy Science, Vol.96, pp.1984-1996, 2013.

[18] A. R. Abdelgawad, B. Guamis, M. Castillo, "Using a fiber optic sensor for cutting time prediction in cheese manufacture from a mixture of cow, sheep and goat milk", Journal of Food Engineering, Vol.125, pp.157-168, 2014.

[19] O. Arango, A. J. Trujillo, and M. Castillo, "Predicting coagulation and syneresis parameters of milk gels when inulin is added as fat substitute using infrared light backscatter", Journal of Food Engineering, Vol.157, pp.63-69, 2015.

[20] N. Nicolau, M. Buffa, D. J. OCallaghan, B. Guamis, and M. Castillo, "Estimation of clotting and cutting times in sheep cheese manufacture using NIR light backscatter", Dairy Science and Technology, Vol.95, pp.495-507, 2015.

[21] L. Benguigui, J. Emery, D. Durand, and J. P. Busnel, "Ultrasonic study of milk clotting", Lait, Vol.74, pp.197-206, 1994.

[22] C. Ay, and S. Gunasekaran, "Ultrasonic attenuation measurements for estimating milk coagulation time", Transactions of the ASAE, Vol.37, pp.857-862, 1994.

[23] S. Gunasekaran, and C. Ay, "Milk coagulation cut-time determination using ultrasonics", 
Journal of Food Process Engineering, Vol.19, pp.63-73, 1996.

[24] F. Bakkali, A. Moudden, B. Faiz, A. Amghar, M. Espinosa, G. Maze, and M. F. Akhnak, "Ultrasonic measurement of milk coagulation time", Measurement Science and Technology, Vol.12, pp.2154-2159, 2001.

[25] G. Nassar, B. Nongaillard, and Y. Noel, "Study by ultrasound of the impact of technological parameters changes in the milk gelation process", Journal of Food Engineering, Vol.63, pp.229236, 2004.

[26] N. Taifi, F. Bakkali, B. Faiz, M. Moudden, G. Maze, and D. Dcultot, "Characterization of the syneresis and the firmness of the milk gel using an ultrasonic technique", Measurement Science and Technology, Vol.17, pp. 281-287, 2006.

[27] A. B. Koc, and B. H. Ozer, "Non-destructive monitoring of renetted whole milk during cheese manufacturing", Food research international, Vol.41, pp.745-750, 2008.

[28] M. Derra, F. Bakkali, A. Amghar and H. Sahsah, "Prediction of milk coagulation time using an ultrasonic experimental and theoretical method based on Argand diagram", Food Measure, DOI : 10.1007/s11694-017-9567-5, 2017.

[29] V. Buckun, and S. Cormor, "High resolution ultrasonic resonator measurements for analysis of liquids", Semin. Food Anal, Vol.4, pp.113130, 1999.

[30] R. A. Kline, "Measurement of attenuation and dispersion using an ultrasonic spectroscopy technique", J. Acoust. Soc. Am, Vol.76, pp.498504, 1984.

[31] D. K. Mak, "Ultrasonic phase velocity measurement incorporating couplant correction", Br. J. Non-Destruct. Test, Vol.35, pp 443-449, 1993.

[32] S. Akker, J. Arman, and P. Mouge, "Ondes ultrasonores dans des plaques: application à l'étude des milieux bicouches", Acoustica, Vol.77, pp.232-239, 1992.

[33] O. Lenoir, J. L. Izbicki, P. Rembert, G. Maze, and J. Ripoche, "Acoustic scattering from an immersed plane multilayer: application to the inverse problem", J. Acoust. Soc. Am, Vol.91, pp.601-612, 1992.

[34] D. K. Mak, "Comparison of various methods for the measurement of reflection coefficient and ultrasonic attenuation", Br. J. Non-Destruct. Test, Vol.33, pp. 441-449, 1991. 